\title{
Design of Nonuniform Near Allpass Complementary FIR Filters via a Semi-infinite Programming Technique
}

\author{
Charlotte Yuk-Fan Ho, Member, IEEE, Bingo Wing-Kuen Ling, Yan-Qun Liu, Peter Kwong-Shun \\ Tam, Member, IEEE, Kok-Lay Teo, Senior Member, IEEE
}

\begin{abstract}
In this paper, we consider the problem of designing a set of nonuniform near allpass complementary FIR filters. This problem can be formulated as a quadratic semi-infinite programming problem, where the objective is to minimize the sum of the ripple energy for the individual filters, subject to the passband and stopband specifications as well as to the allpass complementary specification. The dual parameterization method is used for solving the linear quadratic semi-infinite programming problem.
\end{abstract}

Index Terms - allpass complementary FIR filters, nonuniform frequency bands, semi-infinite programming, dual parameterization.

\section{INTRODUCTION}

I $\mathrm{T}$ is well known that perfect signal reconstruction can be achieved simply by adding all subband signals, if a set of allpass complementary filters is employed in the analysis procedure [1]. Thus, the use of these filters can save substantial implementation cost (see, [1], [4]-[9]). For this reason, it has found many applications in different engineering disciplines, such as in sigma-delta A/D converter designs [2], audio coders [3], speech scramblers [3] and loudspeaker crossovers [5]-[6].

The most common existing design methods of complementary filters are based on the interconnections of some allpass functions [4]-[9]. Although the designed filters are robust to coefficient quantization, these methods are developed

Manuscript received August 7, 2003; revised November 26, 2003. This work was substantially supported by a research grant (project number G-YD26) from the Hong Kong Polytechnic University, the Centre for Multimedia Signal Processing, The Hong Kong Polytechnic University, the CRGC grant (project number PolyU 5101\01E) from the Research Grants Council of Hong Kong, as well as a research grant from Australian Research Council. The associate editor coordinating the review of this manuscript and approving it for publication was Dr. Henrique Malvar.

Charlotte Yuk-Fan Ho is with the Department of Electronic Engineering, Queen Mary, University of London, Bingo Wing-Kuen Ling is with the Department of Electronic Engineering, King's College London, Yan-Qun Liu is with the Department of Mathematics and Statistics, Royal Melbourne Institute of Technology, Peter Kong-Shun Tam is with the Department of Electronic and Information Engineering, and Kok-Lay Teo is with the Department of Applied Mathematics and Centre for Multimedia Signal Processing, Department of Electronic and Information Engineering. (e-mail: wing-kuen.ling@kcl.ac.uk).

Publisher Item Identifier based on the uniform or tree-structured complementary filters. Moreover, these methods are still relying on some unconstrained optimization routines [4]-[5] or incorporating some standard filters [6]-[9], such as elliptic filters, Chebyshev filters and Butterworth filters in a somewhat adhoc manner; so they cannot be applied to minimize the overall passband and stopband ripples.

Since the uniform complementary filters sometimes cannot satisfy certain specifications (see, for example, in audio applications [3]), in these cases, a set of nonuniform allpass complementary filters is preferred. To address these problems, a cosine modulation approach [15] was proposed. Although the formulation in [15] could control the amount of passband and stopband ripples of the prototype filter, the allpass complementary specification for the overall structure may not be satisfied. Moreover, as the optimization problem formulated in [15] is not a convex problem, the quasi Netwon algorithm only gives the local optimal solution, which depends on the initial condition selected.

To address this problem, in this paper, the exact allpass complementary requirement is relaxed to a near allpass complementary requirement. This near allpass complementary specification as well as the passband and stopband specifications for the individual filters are together expressed as inequality continuous constraints in the frequency domain. Our objective is to minimize the sum of the ripple energy for the individual filters, subject to those inequality continuous constraints. The optimal near allpass complementary filter design problem is formulated as a quadratic semi-infinite programming problem. Two common approaches to tackle this problem are the discretization method followed by the semi-definite programming [10] and the application of the Remez-exchange algorithm [11]. However, these methods are not effective for the nonuniform near allpass complementary FIR filter design. For the discretization method followed by the semi-definite programming, it is not guaranteed that the constraints will be satisfied in between the discretization points; although the deviation from the constraints can be reduced by increasing the number of discretization points, increasing the number of constraints will aggravate the complexity of the problem. For the Remez-exchange algorithm, the solution obtained converges to the global optimal solution if and only if 
there are $\mathrm{N}+1$ local extrema with alternating signs $(\mathrm{N}$ is the order of the filters) and these local extrema are all equal in absolute value [11]; although this necessary and sufficient condition is satisfied for the equiripple filter design problem, this is not satisfied for the nonuniform near allpass complementary FIR filter design problem, and so the overall performance of the filter banks would not be optimized, and hence, we cannot guarantee the existence of the optimal solution. On the other hand, for the dual parameterization approach [12]-[14], the semi-infinite programming problem is reduced to a sequence of approximating sub-problems followed by a non-linear finite programming problem. Each of the approximating sub-problems can be readily solved using quadratic programming. The finite non-linear program's global solution can then be obtained from the approximate solution. If the feasible set is nonempty, then an optimal solution is guaranteed no matter what the initial condition is. Besides, as the constraint is expressed as a matrix inequality, it is very flexible to include the nonuniform near allpass complementary constraint or other constraints in the design problem by increasing the number of rows in the corresponding matrix and vector. Furthermore, as we have developed an efficient algorithm for implementing this dual parameterization method [12]-[14], in which the algorithm only involves initialization, computing the local minimum and computing the global minimum, so it is simple to use.

The rest of the paper is organized as follows. In Section II, we formulate the design problem as a linear quadratic semi-infinite programming problem. In Section III, design examples are presented and solved. Section IV concludes the paper.

\section{PROBlem Formulation}

To avoid phase distortions, we assume that the frequency responses of the filters are symmetric and can be expressed as:

$H_{i}(\omega)=a_{i, 0}+2 \sum_{k=1}^{N_{i}} a_{i, k} \cos (k \omega)$, for $i=0,1, \cdots, N-1$,

where $2 N_{i}+1$ is the order of the filter $H_{i}(\omega)$, and $a_{i, k}$, for $i=0,1, \cdots, N-1$, are the real coefficients to be designed. Let the passbands and stopbands of $H_{i}(\omega)$ be, respectively, denoted by $B_{i}^{p}$ and $B_{i}^{s}$, for $i=0,1, \cdots, N-1$. Assume that the desired frequency responses are, for $i=0,1, \cdots, N-1$ :

$$
H_{d_{i}}(\omega)= \begin{cases}1 & \omega \in B_{i}^{p} \\ 0 & \omega \in B_{i}^{s}\end{cases}
$$

Let

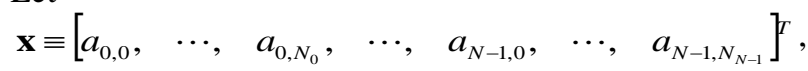

where the superscript ${ }^{T}$ denotes the transpose. The sum of the ripple energy for the individual filter in the passband and stopband can be expressed as:

$$
J(\mathbf{x}) \equiv \sum_{i=0}^{N-1} \int_{B_{i}^{p} \cup B_{i}^{s}}\left|H_{i}(\omega)-H_{d_{i}}(\omega)\right|^{2} d \omega .
$$

Using an argument similar to that given in Appendix B of [14], we can show that there exist a positive definite matrix $\mathbf{Q}$, a vector $\mathbf{b}$ and a scalar $p$ such that:

$$
J(\mathbf{x})=\frac{1}{2} \mathbf{x}^{T} \mathbf{Q} \mathbf{x}+\mathbf{b}^{T} \mathbf{x}+p .
$$

For details, see Appendix B of [14].

Next, we consider the specifications, expressed as a set of the continuous inequality constraints, to be imposed. Let the bounds on the passband and stopband ripples of the filter be, respectively, $\delta_{p_{i}}$ and $\delta_{s_{i}}$, for $i=0,1, \cdots, N-1$. Then the continuous inequality constraints are:

$\left|H_{i}(\omega)-H_{d_{i}}(\omega)\right| \leq \delta_{p_{i}}$, for $\omega \in B_{i}^{p}$ and for $i=0,1, \cdots, N-1$,

and

$\left|H_{i}(\omega)-H_{d_{i}}(\omega)\right| \leq \delta_{s_{i}}$, for $\omega \in B_{i}^{s}$ and for $i=0,1, \cdots, N-1$.

By shifting and scaling the frequency bands, all the filters with different bandwidths can be transformed into a lowpass filter with the same bandwidth. Hence, by combining those constraints in a matrix formulation, the constraints can be further expressed as:

$\widetilde{\mathbf{A}}_{f}(\omega) \mathbf{x} \leq \widetilde{\mathbf{c}}_{f}(\omega)$, for $\omega \in B_{0}^{p}$,

where $\quad \tilde{\mathbf{A}}_{f}(\omega)=\operatorname{diag}\left(\mathbf{A}_{0}(\omega), \mathbf{A}_{1}(\omega), \cdots, \mathbf{A}_{N-1}(\omega)\right) \quad$ and $\widetilde{\mathbf{c}}_{f}(\omega)=\left[\begin{array}{llllll}\boldsymbol{\delta}_{0}, & \boldsymbol{\delta}_{1}, & \cdots, & \boldsymbol{\delta}_{N-2}, & \boldsymbol{\delta}_{N-1}\end{array}\right]^{T}$ for $\omega \in B_{0}^{p}, \quad$ in which $\mathbf{A}_{i}(\omega)$ and $\boldsymbol{\delta}_{i}$ consist of respectively, cosine functions and the specifications for the passband and stopband ripples. Now define the allpass complementary constraint as:

$\left|\left(\sum_{i=0}^{N-1} H_{i}(\omega)\right)-A(\omega)\right| \leq \delta_{a}$, for $\omega \in[-\pi, \pi]$,

where $A(\omega)$ is an allpass function and $\delta_{a}$ is the upper bound for the absolute difference between the sum of the filter responses and the allpass function. Similarly, the constraint defined in (9) can be expressed as:

$\widetilde{\mathbf{A}}_{r}(\omega) \mathbf{x} \leq \widetilde{\mathbf{c}}_{r}(\omega)$, for $\omega \in B_{0}^{p}$,

where

$$
\hat{\mathbf{A}}_{r}(\omega)=\left[\xi\left(\frac{\omega-\alpha_{N}}{\beta_{N}}\right), \quad-\xi\left(\frac{\omega-\alpha_{N}}{\beta_{N}}\right)\right]^{T}
$$

$\hat{\mathbf{c}}_{r}(\omega)=\left[\delta_{a}+A\left(\frac{\omega-\alpha_{N}}{\beta_{N}}\right), \quad \delta_{a}-A\left(\frac{\omega-\alpha_{N}}{\beta_{N}}\right)\right]^{T}$, in which $\xi(\omega), \alpha_{N}$ and $\beta_{N}$ are respectively, the kernels of the filters, the amount of frequency shift and the scaling factor for the bandwidth. Hence, the near allpass complementary FIR filter design problem can be formulated as the following linear quadratic semi-infinite programming problem:

\section{Problem $(\mathrm{P})$}

$\min _{\mathbf{x}} \quad J(\mathbf{x})=\frac{1}{2} \mathbf{x}^{T} \mathbf{Q} \mathbf{x}+\mathbf{b}^{T} \mathbf{x}+p$,

subject to $\mathbf{g}(\mathbf{x}, \omega)=\mathbf{A}(\omega) \mathbf{x}-\mathbf{c}(\omega) \leq \mathbf{0}$, for $\omega \in B_{0}^{p}$,

where $\mathbf{A}(\omega)=\left[\begin{array}{c}\hat{\mathbf{A}}_{f}(\omega) \\ \hat{\mathbf{A}}_{r}(\omega)\end{array}\right]$ and $\mathbf{c}(\omega)=\left[\begin{array}{l}\hat{\mathbf{c}}_{f}(\omega) \\ \hat{\mathbf{c}}_{r}(\omega)\end{array}\right]$.

For a fixed set of upper bound values for the passband and stopband ripples, as well as the allpass complementary error, the design procedure based on the dual parameterization given in 
[12]-[13] is used. The first part of the design procedure is to formulate the design problem as a semi-infinite programming problem, referred to as Problem (P), as described above. Since the algorithm for obtaining the solution of Problem $(\mathrm{P})$ is stated in [12], the next part of the design procedure follows the steps stated in [12]. That is, first initialize a set of parameters, then compute the local minimum of the finite problem. Finally, compute the global minimum by implementing a local search for the finite dual problem, and this global minimum corresponds to the optimal solution of Problem (P).

The nonuniform near allpass complementary FIR filter design problem is formulated as a semi-infinite programming problem, referred to as Problem (P), and we have shown in [12]-[14] that the solution for Problem (P) will converge to an exact optimal solution satisfying all the required specifications if the KKT conditions are satisfied. Thus, the convergence of the dual parameterization method [12]-[14] to an exact optimal solution is guaranteed once the KKT conditions are satisfied. In order to satisfy the KKT conditions, we need to guarantee that the feasible set is nonempty. It is well known in the filter design theory [16] that the magnitude specifications on the passband and stopband will be satisfied if the filter length is long enough. Hence, the feasible solution is nonempty and KKT conditions are satisfied if the filter lengths of the filter are long enough. As a result, the requirement for our algorithm to obtain an exact optimal solution is the sufficiently long filter lengths.

\section{Simulation Results}

For comparison, consider the design example shown in [9]. The specifications of the filters are as follows: (i) the cutoff frequency of the lowpass filter is $0.2 \pi$, that of the bandpass filter are $0.2 \pi$ and $0.7 \pi$, and that of the highpass filter is $0.7 \pi$; (ii) the passband and stopband ripples of all the filters are bounded by the values of $-0.00043 \mathrm{~dB}$ and $-40 \mathrm{~dB}$, respectively. Assume the transition bandwidths of all the filters be $0.05 \pi$ and the allpass function be $A(\omega)=1$ for $\omega \in[-\pi, \pi]$. Let the maximum allowable allpass complementary error be $\delta_{a}=0.0151$. We start the design of the filters with the filter length $N_{i}=70$ for $i=0,1,2$. The responses of the filters and the allpass complementary error designed via our approach are, respectively, plotted as solid lines in Figure 1 and Figure 2. The stopband ripple of the filters is just bounded by $-42 \mathrm{~dB}$ for the case considered in [9], while it is about $-100 \mathrm{~dB}$ for our case. In order to illustrate the effectiveness of formulating the design problem as a semi-infinite programming problem with the continuous constraint, the responses of the filters and the allpass complementary error designed with the same cost function but without the constraint are also plotted as dotted lines in, respectively, Figure 1 and Figure 2. Although the ripple energy of the filters designed via our approach is higher than that of the unconstrained design, the allpass complementary error of our design is bounded by $-36.4258 \mathrm{~dB}$, and that of the unconstrained design is just bounded by $-34.4933 \mathrm{~dB}$, which does not satisfy the required specification. By using our design, the specification is met and there is $1.9325 \mathrm{~dB}$ improvement.

Consider another design example with the specifications defined as follows: (i) the cutoff frequency of the lowpass filter is $0.5 \pi$, that of the bandpass filter are $0.5 \pi$ and $\frac{5 \pi}{6}$, and that of the highpass filter is $\frac{5 \pi}{6}$; (ii) the passband and stopband ripples of the lowpass filter are bounded by the values of, respectively, 0.0331 and 0.0330 , that of the bandpass are, respectively, 0.0378 and 0.0404 , and that of the highpass filter are, respectively, 0.0313 and 0.0366 ; (iii) the transition bandwidths of all the filters are 0.15 and the allpass function is $A(\omega)=1$ for $\omega \in[-\pi, \pi]$; (iv) the maximum allowable allpass complementary error is $\delta_{a}=0.0189$. We start the design of the filters with the filter length $N_{i}=20$ for $i=0,1,2$. The responses of the filters and the allpass complementary error designed via our approach and the unconstrained approach are plotted as, respectively, solid lines and dotted lines in Figure 3 and Figure 4. By magnifying the band edges of the filters and the allpass complement error as shown in Figure 4, it can be seen clearly that the unconstrained case does not satisfy some of the passband and stopband specifications as well as the allpass complementary specification. On the other hand, the filters designed by our proposed method satisfy all the specifications. The maximum allpass complementary error designed by our proposed method is bounded by $-34.4904 \mathrm{~dB}$, which is $1.0797 \mathrm{~dB}$ lower than that obtained using the unconstrained optimization method.

\section{CONCLUSION}

The main contribution of this paper is the formulation of a nonuniform near allpass complementary FIR filters design problem as a linear quadratic semi-infinite programming problem. The dual parameterization algorithm was used to solve this problem. The algorithm can find an exact optimal solution that satisfies the passband and stopband specifications and meets the allpass complementary specification if the solution exists.

\section{ACKNOWLEDGEMENT}

The work obtained in this paper was supported by a research grant (project number G-YD26) from the Hong Kong Polytechnic University, the Centre for Multimedia Signal Processing, The Hong Kong Polytechnic University, the CRGC grant (project number PolyU 5101\01E) from the Research Grants Council of Hong Kong, as well as a research grant from Australian Research Council.

\section{REFERENCES}

[1] S. R. Pillai and G. H. Allen, "Higher order complementary filters," IEEE Transactions on Signal Processing, vol. 42, no. 10, pp. 2848-2851, 1994.

[2] A. Petraglia and S. K. Mitra, "High-speed A/D conversion incorporating a QMF bank," IEEE Transactions on Instrumentation and Measurement, vol. 41, no. 3, pp. 427-431, 1992.

[3] R. V. Cox, "The design of uniformly and nonuniformly spaced 
pseudoquadrature mirror filters," IEEE Transactions on Acoustics, Speech, and Signal Processing, vol. ASSP-34, no. 5, pp. 1090-1096, 1986.

[4] H. Johansson and T. Saramäki, "A class of complementary IIR filters," IEEE International Symposium on Circuits and Systems, ISCAS, vol. 3, pp. 299-302, 1999.

[5] P. A. Regalia and S. K. Mitra, "A class of magnitude complementary loudspeaker crossovers," IEEE Transactions on Acoustics, Speech and Signal Processing, vol. ASSP-35, no. 11, pp. 1509-1516, 1987.

[6] S. K. Mitra, Y. Neuvo and P. P. Vaidyanathan, "Complementary IIR digital filter banks," IEEE International Conference on Acoustics, Speech and Signal Processing, ICASSP, vol. 10, pp. 529-532, 1985.

[7] P. A. Regalia, S. K. Mitra and P. P. Vaidyanathan, "The digital all-pass filter: a versatile signal processing building block," Proceedings of the IEEE, vol. 76, no. 1, pp. 19-37, 1988.

[8] P. P. Vaidyanathan, P. A. Regalia and S. K. Mitra, "Design of doubly-complementary IIR digital filters using a single complex allpass filter, with multirate applications," IEEE Transactions on Circuits and Systems, vol. CAS-34, no. 4, pp. 378-389, 1987.

[9] P. A. Regalia, S. K. Mitra, P. P. Vaidyanathan, M. K. Renfors and Y. Neuvo, "Tree-structured complementary filter banks using all-pass sections," IEEE Transactions on Circuits and Systems, vol. CAS-34, no. 12, pp. 1470-1484, 1987.

[10] W. S. Lu, "A unified approach for the design of 2-D digital filters via semidefinite programming," IEEE Transactions on Circuits and Systems - I: Fundamental Theory and Applications, vol. 49, no. 6, pp. 814-826, 2002.

[11] E. Z. Psarakis and G. V. Moustakides, "A robust initialization scheme for the Remez exchange algorithm," IEEE Signal Processing Letters, vol. 10, no. 1, pp. 1-3, 2003.

[12] Y. Liu, C. H. Tseng and K. L. Teo, "A unified quadratic semi-infinite programming approach to time and frequency domain constrained digital filter design," Communications in Information and Systems, vol. 2, no. 4, pp. 399-410, 2002.

[13] Y. Liu, K. L. Teo and S. Ito, "Global optimization in quadratic semi-infinite programming," Computing, vol. 15, pp. 119-132, 2001.

[14] H. H. Dam, K. L. Teo, S. Nordebo and A. Cantoni, "The dual parameterization approach to optimal least square FIR filter design subject to maximum error constraints," IEEE Transactions on Signal Processing, vol. 48, no. 8, pp. 2314-2320, 2000

[15] S. Wada, H. Yagi and T. Saito, "A flexible design of nonuniformly oversampled multirate filter banks," IEEE International Symposium on Circuits and Systems, ISCAS, vol. 4, pp. 838-841, 1996.

[16] P. P. Vaidyanathan, Multirate systems and filter banks, Englewood Cliffs, NJ: Prentice-Hall, 1993.
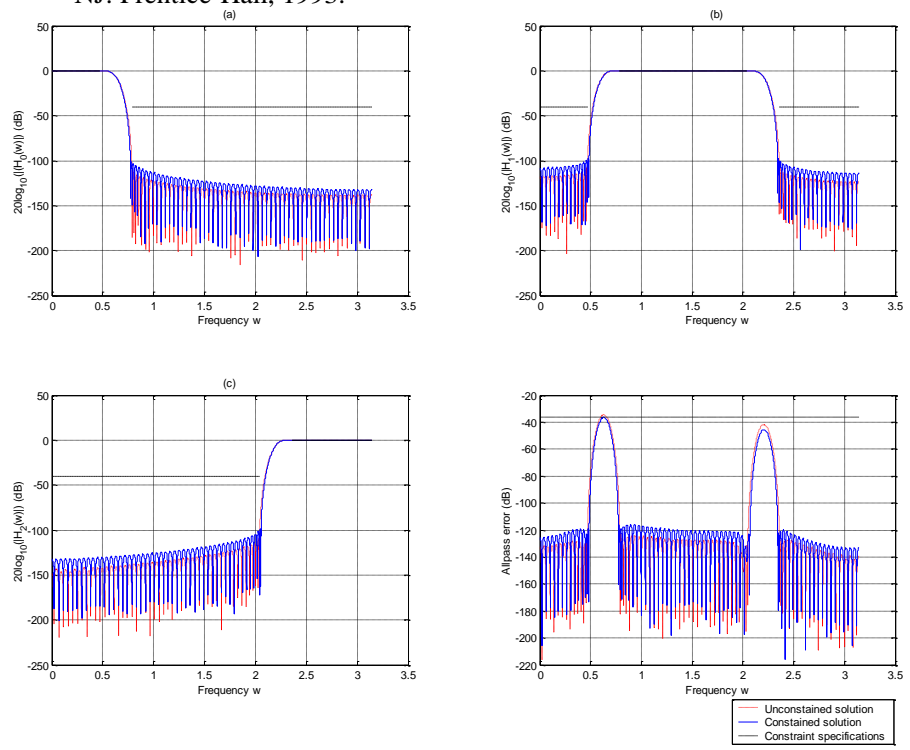

Figure 1: (a)-(c) The filter responses obtained using the unconstrained technique and our proposed constrained method. (d) The allpass complementary errors obtained using the unconstrained technique and our proposed constrained method.

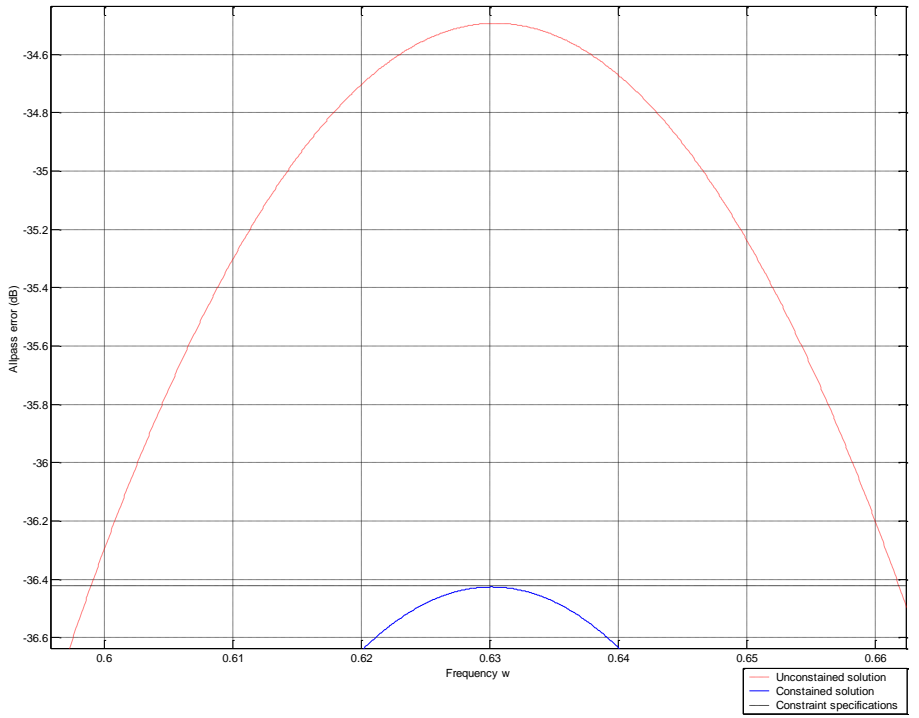

Figure 2: Zoom-in for the allpass complementary errors obtained using the unconstrained technique and our proposed constrained method.
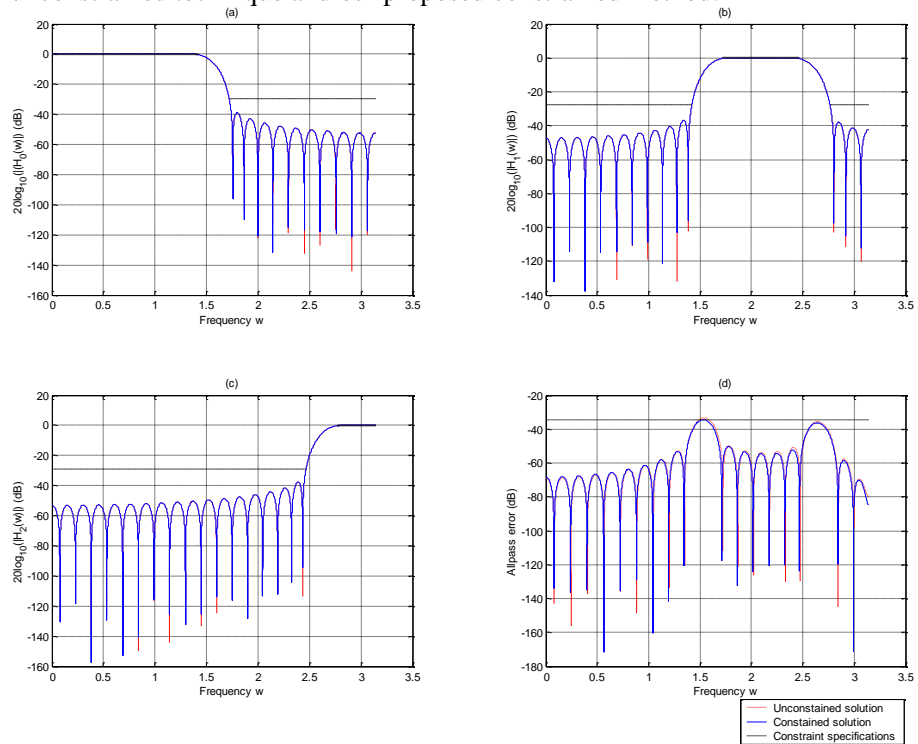

Figure 3: (a)-(c) The filter responses obtained using the unconstrained technique and our proposed constrained method. (d) The allpass complementary errors obtained using the unconstrained technique and our proposed constrained method. 

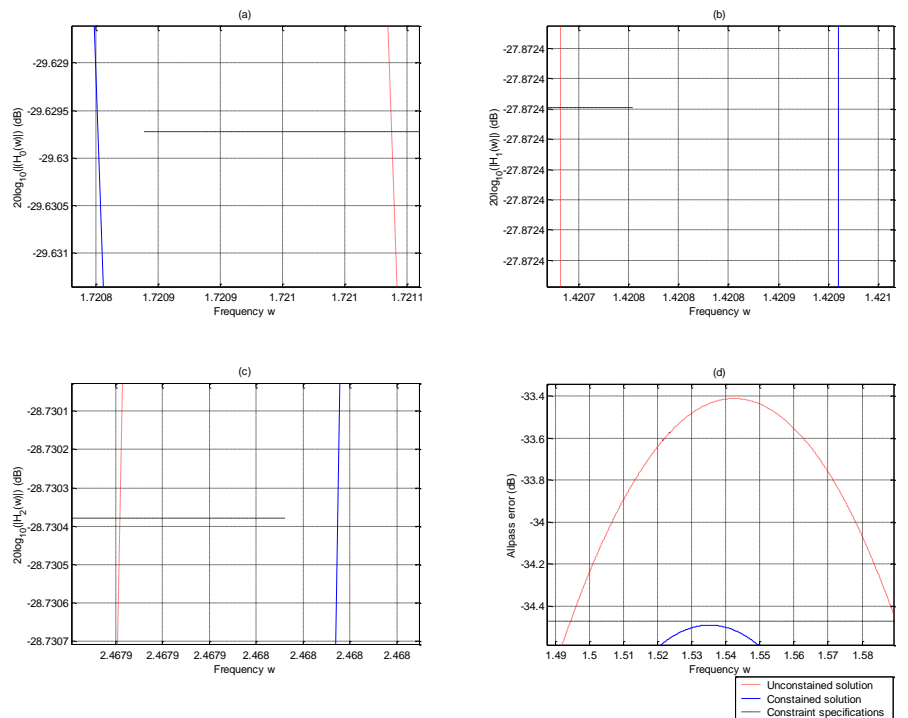

Figure 4: (a)-(c) Zoom-in for the filter responses obtained using the unconstrained technique and our proposed constrained method. (d) Zoom-in for the allpass complementary errors obtained using the unconstrained technique and our proposed constrained method. 